Joseph Felix KouedJI MonthÉ ${ }^{1}$

Annie-Claude PIAL ${ }^{2}$

Guy Merlin Nguenang ${ }^{3}$

Ghislain Aimé Fomou NYAMSI ${ }^{1}$

1 Université de Yaoundé I

Faculté des sciences

Laboratoire de botanique-écologie

BP 812, Yaoundé

Cameroun

2 Université de Yaoundé I

Faculté des sciences

Département de biologie

et physiologie végétales

BP 812, Yaoundé

Cameroun

${ }^{3} \mathrm{GIZ} /$ ProPSFE/Antenne

de la Région de l'Est

BP 07, Bertoua

Cameroun

\title{
Gestion participative des forêts : évaluation de l'efficacité des Comités paysans-forêts dans l'Est-Cameroun
}

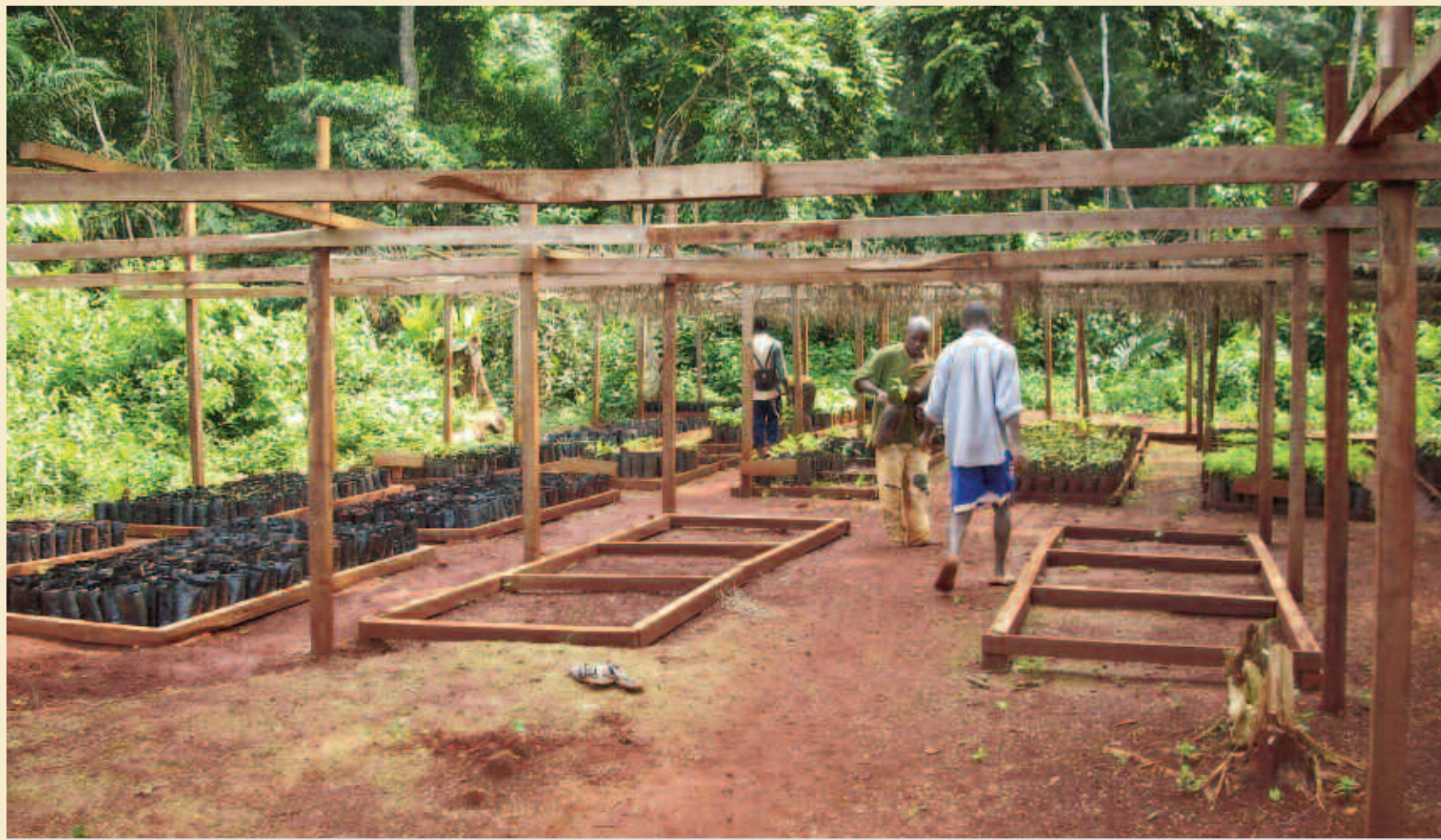

Photo 1.

Une pépinière du Comité paysans-forêts Nampella-Nlong, située à Mparo.

Photo J. F. Kouedji Monthe. 


\section{RÉSUMÉ}

\section{GESTION PARTICIPATIVE DES FORÊTS : ÉVALUATION DE L'EFFICACITÉ DES COMITÉS PAYSANS-FORÊTS DANS L'EST-CAMEROUN}

Le secteur forestier camerounais joue un rôle très important dans l'économie nationale et assure un ensemble de fonctions sociales et environnementales. Dans le souci de gérer ce secteur de manière durable et participative, la loi forestière, fruit de l'opérationnalisation des recommandations du sommet de Rio de Janeiro, prévoit l'implication des Comités paysans-forêts (CPF), structures représentatives des populations locales, dans la gestion forestière. Cet article se propose de tirer les leçons de la gestion participative des forêts à travers l'évaluation de l'efficacité des CPF de deux départements de la région de l'Est-Cameroun, la Boumba et Ngoko et la Kadey. L'évaluation de ces CPF a été conduite sur la base des dispositions légales énoncées dans la décision $n^{\circ}$ 1354/D/MINEF/CAB du 26 novembre 1999, de laquelle six critères d'évaluation et 20 indicateurs de performance ont été tirés. Les données collectées sur le terrain laissent entrevoir de nombreux dysfonctionnements : inexistence des CPF dans certaines localités sujettes à l'exploitation forestière, absence de document normatif et statutaire pour la majorité des CPF, insuffisance des ressources financières autonomes, faible collaboration avec les autres acteurs de la gestion forestière et déséquilibre dans la représentativité des différentes composantes sociales au sein des bureaux. L'analyse des écarts montre que les niveaux de conformité des CPF avec les exigences légales dans les départements ciblés sont très faibles. Aucun critère n'est conforme et $65 \%$ des indicateurs présentent des niveaux de conformité nuls. En définitive, les objectifs assignés aux CPF ne sont pas atteints, ce qui met en évidence leur inefficacité. Des pistes d'amélioration sont proposées autour de l'information, de l'autonomisation et de la formation des CPF.

Mots-clés : gestion participative, forêt, comité paysans-forêts, Cameroun.

\section{PARTICIPATORY FOREST MANAGEMENT: A PERFORMANCE ASSESSMENT OF SMALLHOLDER-FORESTER COMMITTEES IN EASTERN CAMEROON}

Cameroon's forest sector makes a substantial contribution to the national economy and also fulfils a wide range of social and environmental functions. In order to establish sustainable and participatory management of the forest sector, the forest legislation adopted in line with the Rio Summit recommendations provides for the involvement of SmallholderForester Committees (CPF) that must be representative of local populations. This article aims to draw lessons for participatory forest management from an assessment of the effectiveness of CPFs in two districts (Boumba-Ngoko and Kadey) in eastern Cameroon. The CPF assessment was conducted in accordance with the legal provisions set out in Decision No $1354 / D / M I N E F / C A B$ dated November $26^{\text {th }} 1999$, from which 6 evaluation criteria and 20 performance indicators were developed. Data collected in the field reveal dysfunctions in many areas: nonexistence of CPFs in some heavily logged areas, absence of statutory and compliance documents in many CPFs, inadequacy of autonomous funding, lack of cooperation with other forest management partners and unequal representation of social groups in committee decisions. Gap analysis shows that CPF compliance with legal requirements in the relevant districts is very low. None of the criteria are met and compliance is nil for $65 \%$ of the indicators. Clearly, the objectives assigned to the CPFs are not being achieved, revealing their ineffectiveness. Proposed avenues for improvement focus on information, empowerment and training for CPFs.

Keywords: participatory management, forest, smallholder-forester committees, Cameroon.

\section{MANEJO PARTICIPATIVO DE BOSQUES: EVALUACIÓN DE LA EFICACIA DE LOS COMITÉS CAMPESINOS-BOSQUES EN EL ESTE DE CAMERÚN}

El sector forestal camerunés desempeña un papel muy importante en la economía nacional y presta una serie de funciones sociales y ambientales. Para manejar dicho sector de modo sostenible y participativo, la ley forestal en la que se plasman las recomendaciones de la cumbre de Río de Janeiro prevé la implicación de estructuras representativas de las poblaciones locales, los Comités CampesinosBosques (CCB), en el manejo forestal. Este artículo se propone sacar enseñanzas del manejo participativo de los bosques mediante la evaluación de la eficacia de los CCB de dos departamentos de la Región del Este de Camerún: Boumbaet-Ngoko y Kadey. La evaluación de estos CCB se realizó basándose en las disposiciones legales expresadas en la decisión No 1354/D/MINEF/CAB del 26 de noviembre de 1999 , extrayéndose seis criterios de evaluación y 20 indicadores de rendimiento. Los datos obtenidos en campo permiten apreciar numerosas deficiencias: inexistencia de CCB en algunas localidades sometidas a aprovechamiento forestal, ausencia de documento normativo y jurídico en la mayoría de CCB, insuficiencia de recursos financieros autónomos, baja colaboración con los demás actores del manejo forestal y desequilibrio en la representatividad de los distintos componentes sociales en las oficinas. El análisis de las deficiencias muestra que los niveles de conformidad de los CCB con las exigencias legales en estos departamentos son muy bajos. No se cumple completamente ningún criterio y el $65 \%$ de los indicadores muestra niveles de conformidad nulos. En conclusión, no se logran los objetivos atribuidos a los CCB, poniéndose de manifiesto su ineficacia. Se realizan propuestas de mejora centradas en la información, autonomización y formación de los CCB.

Palabras clave: manejo participativo, bosque, comité campesinos-bosque, Camerún. 


\section{Introduction}

Avec un peu plus de 17 millions d'hectares de forêts denses humides, le Cameroun figure parmi les quatre principaux pays forestiers du Bassin du Congo (De Wasseige et al., 2010). Le secteur forestier est très important pour son économie ; sa valeur ajoutée a contribué à hauteur de 2,76\% au produit intérieur brut entre 2008 et 2010, tandis que les exportations ont représenté en moyenne 218,5 milliards de francs CFA par an de 2008 à 2009 (Eba'a Atyi et al., 2013). Malgré son rôle dans le développement du Cameroun, la gestion forestière continue de faire face à de nombreux défis, notamment la révision de son cadre juridique et institutionnel.

Le nouveau cadre adopté en 1994 consacre la gestion participative, matérialisée par la prise en compte des populations locales dans la gestion des ressources forestières. Cette innovation majeure découle des recommandations du sommet de Rio de Janeiro. La gestion participative des forêts permet aux populations concernées par l'exploitation du domaine forestier d'en être les acteurs, au même titre que l'État et les exploitants. Dans le domaine forestier non permanent, l'État camerounais octroie entièrement les droits d'exploitation aux communautés villageoises, à travers les forêts communautaires. Dans le domaine forestier permanent, il confie aux populations locales et autochtones les droits de consultation, de négociation et de participation dans la gestion des ressources forestières, ceci à travers divers organes : Comités de valorisation des ressources fauniques (Covaref), Comités riverains (CR) et Comités paysans-forêts (CPF).

Les CPF sont les intermédiaires privilégiés entre l'administration forestière et les populations locales. Ce sont des organes de consultation, de négociation et de participation des populations locales à la gestion des ressources forestières dans les forêts du domaine permanent. Plusieurs missions leur sont attribuées par la décision n $1354 / D / M I N E F /$ CAB $^{1}$ du 26 novembre 1999, notamment l'information, l'animation et la sensibilisation des populations villageoises, mais aussi la participation à l'élaboration des plans de gestion forestière, à l'exécution des travaux en forêt, à la surveillance et au contrôle de l'exploitation forestière illégale.

Les CPF sont composés de huit membres librement élus par l'ensemble des villageois sur la base de critères bien établis (honnêteté, dynamisme, disponibilité et engagement pour l'intérêt général de la communauté). Les membres des $\mathrm{CPF}$, dont le mandat est de trois ans renouvelables, doivent se distinguer par leur esprit de dialogue et d'écoute envers les populations, et par la transmission de l'information. Ils se réunissent au moins quatre fois par an sur convocation de leur président. Afin de faciliter les liens de communication entre les CPF et les autres intervenants dans la gestion forestière (organes de l'administration forestière, exploitants forestiers, collectivités territoriales décentralisées), des réunions peuvent être programmées dans l'optique de faire circuler l'information et planifier les activités à venir.

Après plus d'une décennie de fonctionnement, il est opportun de procéder à une évaluation de l'action des CPF, gages de l'implication effective des populations locales dans la gestion des ressources forestières. La présente étude se propose d'évaluer l'efficacité des CPF de deux départements de la région de l'Est du Cameroun, sur la base des dispositions légales, et de contribuer à l'amélioration de leur efficacité.

${ }^{1}$ MINEF/CAB : ministère de l'Environnement et des Forêts/Cabinet.

\section{Matériel et méthodes}

\section{Site d'étude}

L'étude a pour cadre les départements de la Boumba et Ngoko (de $1^{\circ} 80^{\prime}$ à $4^{\circ} 05^{\prime}$ de latitude Nord et de $14^{\circ} 00^{\prime}$ à $16^{\circ} 00^{\prime}$ de longitude Est) et de la Kadey (de $3^{\circ} 40^{\prime}$ à $5^{\circ} 20^{\prime}$ de latitude Nord et de $13^{\circ} 30^{\prime}$ à $15^{\circ} 20^{\prime}$ de longitude Est). Le choix de ces deux départements est motivé par la présence depuis les années 1950 des sociétés d'exploitation forestière, la proportion des recettes générées dans le budget de l'État, l'importance des fonctions socio-économiques et environnementales de la forêt, et les expériences participatives initiées par plusieurs acteurs.

Le domaine forestier permanent est constitué d'aires protégées, de forêts de production à utilisations multiples subdivisées en Unités forestières d'aménagement (UFA) et en Forêts communales (FC), sur lesquelles sont assises les Zones d'intérêt cynégétique (ZIC). On dénombre 22 UFA, 4 FC et 10 ZIC dans le département de la Boumba et Ngoko, et 10 UFA et 1 FC dans la Kadey (figures 1 et 2).

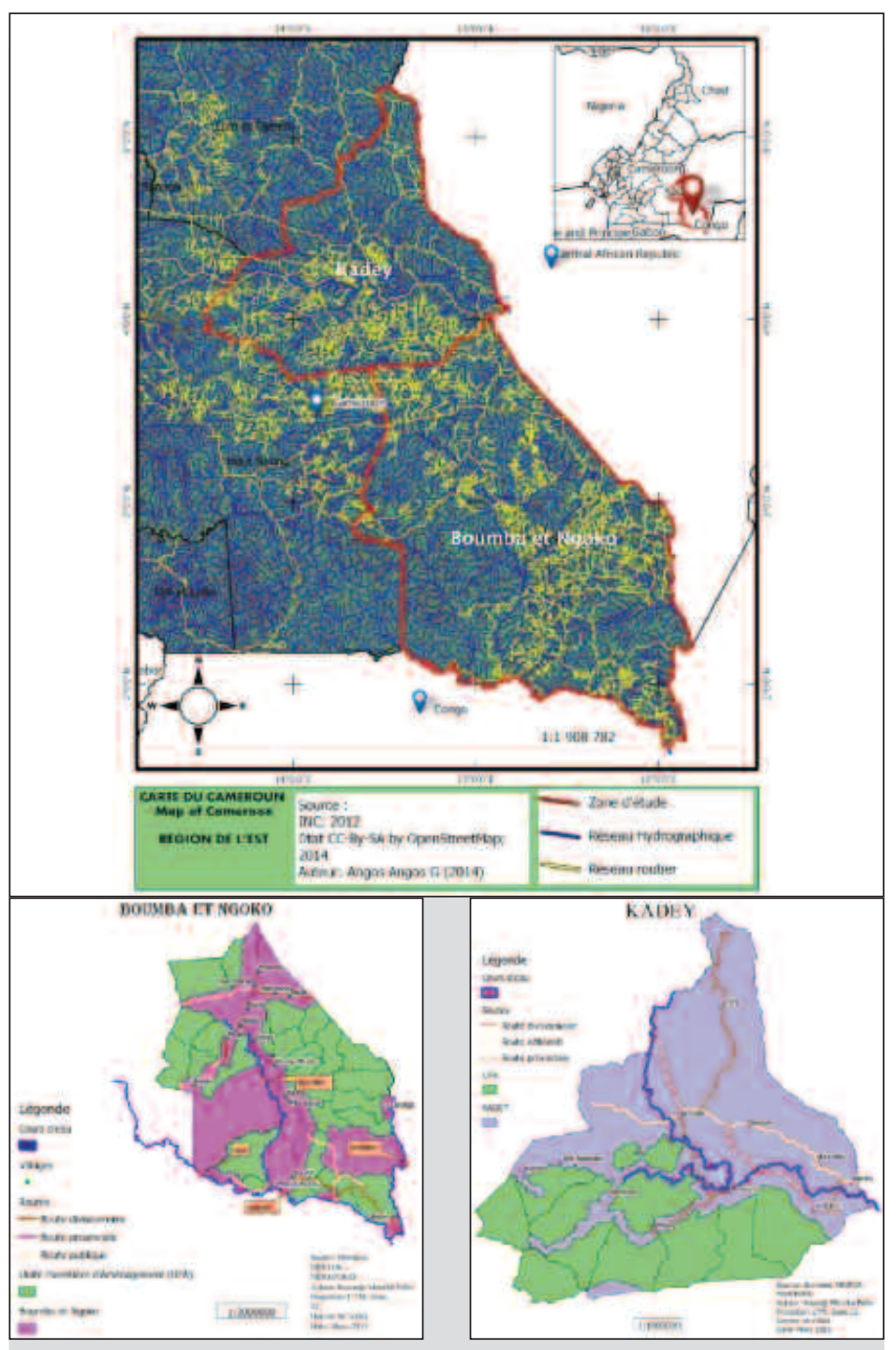

Figures 1 et 2

Localisation des départements de la Boumba et Ngoko et de la Kadey au Cameroun. 


\section{Choix de l'échantillon}

Un recensement exhaustif des CPF de notre zone d'étude a été réalisé à partir de la documentation mise à notre disposition par l'administration forestière, les sociétés forestières et les organisations non gouvernementales (ONG) locales ayant facilité le processus de mise en place de ces structures. Parmi les 53 CPF dénombrés, 33 (16 pour la Boumba et Ngoko et 17 pour la Kadey) ont été retenus pour constituer notre échantillon. Ce choix a reposé sur la représentativité des titres forestiers (UFA et FC couvertes par les (PF) et des unités administratives, mais aussi le nombre de villages couverts par le CPF ou sa zone d'emprise.

\section{Collecte des données}

\section{Collecte des données primaires}

La collecte des données primaires a bénéficié de l'appui d'un facilitateur local chargé de la traduction, et a été réalisée en recourant à plusieurs outils :

- les questionnaires d'enquête, présentés uniquement aux membres des CPF en tenant compte du genre et de la minorité baka, et de fait soumis à un total de 129 personnes (tableau l);

- les interviews semi-structurées, relevant d'un guide d'entretien et conduites auprès des personnes-ressources référencées dans les groupes d'acteurs majeurs (exploitants forestiers, mairie, administration des forêts) ; les principaux thèmes portaient sur le rôle de la personne interrogée dans la mise en place et l'accompagnement des CPF, ses attentes vis-à-vis des CPF, et les potentiels axes de redynamisation des CPF qu'elle identifiait ; au total 28 interviews semistructurées ont été réalisées ;

- les réunions de type «focus group », utilisées pour les CPF riverains de deux titres d'exploitation détenus par des concessionnaires différents ; sept réunions ont été organisées, avec une moyenne de participation de six personnes par CPF, et ont porté sur la nature des rapports entretenus entre les CPF et les autres acteurs de la gestion forestière, de même que sur les principales difficultés rencontrées par les membres des CPF dans le cadre de leurs missions; - les observations directes, permettant de faire émerger de nouvelles questions, de recueillir des informations supplémentaires et d'améliorer ainsi le formulaire d'enquête pour mieux le focaliser sur l'efficacité des CPF.

\section{Collecte des données secondaires}

La collecte des données secondaires a été menée à partir d'informations tirées d'ouvrages, de journaux et d'articles scientifiques présentant un intérêt pour le thème étudié. Elle a eu pour cadre les CPF, les cellules d'aménagement des entreprises forestières, les cellules forestières communales, les services déconcentrés de l'État à l'instar des délégations départementales et des postes de contrôle forestier et de chasse émanant du ministère des Forêts et de la Faune.

\section{Analyse des données}

Une triangulation des différentes données collectées a été effectuée afin d'éliminer les informations non fiables. Les données issues des questionnaires d'enquête ont été dépouillées manuellement, encodées avec le logiciel Excel en prélude à une analyse quantitative.

\section{Évaluation et analyse des écarts aux dispositions officielles}

L'évaluation et l'analyse des écarts aux dispositions officielles ont été réalisées sur la base de la décision n 1354 évoquée plus haut, fixant les procédures de classement des forêts du domaine permanent au Cameroun. Six critères d'évaluation et vingt indicateurs de performance ont été tirés des dispositions de cette décision qui précise le mandat et le fonctionnement des CPF (tableau II).

\section{Résultats}

\section{Organisation et fonctionnement des Comités paysans-forêts}

\section{Recensement des CPF}

Les recherches menées sur le terrain ont permis de dénombrer 25 et 28 CPF respectivement dans la Boumba et Ngoko et la Kadey. Tous ces CPF ont été mis en place entre 2007 et 2011, majoritairement par les concessionnaires d'UFA. Sept l'ont été par la commune de Batouri, qui a bénéficié de l'appui du Centre technique de la forêt communale (CTFC). On observe une forte présence des CPF autour des FC et des UFA attribuées. Certains CPF charnières sont riverains d'au moins deux concessions forestières. La totalité des UFA et FC de la Boumba et Ngoko sont couvertes par les CPF. Par contre, $56 \%$ des UFA et $100 \%$ des FC le sont dans la Kadey.

Tableau I.

Répartition des membres des CPF soumis aux questionnaires d'enquête.

\begin{tabular}{|c|c|c|c|c|c|}
\hline \multirow[t]{2}{*}{ Département } & \multicolumn{2}{|c|}{ Bantous } & \multicolumn{2}{|c|}{ Bakas } & \multirow[t]{2}{*}{ Total } \\
\hline & Hommes & Femmes & Hommes & Femmes & \\
\hline Boumba et Ngoko & 41 & 16 & 09 & 0 & 66 \\
\hline Kadey & 33 & 13 & 14 & 3 & 63 \\
\hline Total & 74 & 29 & 23 & 3 & 129 \\
\hline
\end{tabular}


Tableau II.

Grille de conformité des CPF (cf. décision nº 1354).

Critère 1 Les CPF assurent l'animation et la sensibilisation dans les villages.

Indicateur 1.1 : les CPF organisent des séances de discussion avec les villageois.

Indicateur 1.2 : les CPF participent à la vulgarisation de la loi forestière.

Indicateur 1.3 : les CPF participent à la vulgarisation des Plans d'aménagement (PA).

Indicateur 1.4 : les CPF écoutent les villageois et retransmettent leurs suggestions.

Indicateur 1.5 : les CPF recherchent toute information utile en relation avec l'aménagement des forêts.

Critère 2 Les CPF informent les populations sur l'aménagement des forêts.

Indicateur 2.1 : les CPF organisent des séances d'information dans les villages.

Indicateur 2.2 : les CPF font connaître et expliquent les décisions de l'administration.

Indicateur 2.3 : les CPF informent l'administration sur les initiatives des populations locales.

Critère 3 Les membres des CPF sont directement impliqués dans toutes les étapes de l'élaboration des Plans d'aménagement et des Plans d'opération annuels.

Indicateur 3.1 : les CPF participent à la réalisation de l'enquête socio-économique.

Indicateur 3.2 : les CPF donnent leur avis motivé sur le PA et les plans de gestion des massifs forestiers. Indicateur 3.3 : les CPF participent à la définition des mesures de protection de l'environnement et de la préservation de la diversité biologique de la forêt.

\section{Critère 4 Les CPF participent à l'exécution des travaux en forêt.}

Indicateur 4.1 : les CPF participent au suivi de la bonne exécution du PA.

Indicateur 4.2 : les CPF identifient les ressources humaines et matérielles disponibles au niveau des villages. Indicateur 4.3 : les CPF exécutent ou organisent la réalisation de certains travaux.

Indicateur 4.4 : les CPF participent au suivi des travaux sylvicoles et à leur évaluation technique.

Indicateur 4.5 : les CPF participent aux travaux de délimitation des forêts.

\section{Critère 5 Les CPF participent à la surveillance et au contrôle de l'exploitation forestière illégale.}

Indicateur 5.1 : les CPF organisent des patrouilles de surveillance le long des limites des massifs forestiers. Indicateur 5.2 : les CPF rapportent aux chefs de postes forestiers les cas d'exploitation illégale observés. Indicateur 5.3 : les CPF accompagnent le chef de poste forestier lors des missions de répression.

\section{Critère 6 Les CPF organisent régulièrement des réunions.}

Indicateur 6.1 : les CPF tiennent au moins quatre réunions par an.

La mise en place de ces CPF est l'aboutissement d'un processus dans lequel sont intervenus plusieurs acteurs. Les exploitants forestiers (groupe Sefac, groupe Thanry, groupe Decolvenaere, groupe Rougier), plus ou moins engagés dans le processus de certification forestière, représentent les principaux initiateurs, ayant pris en charge la coordination et le financement du processus. Les ONG impliquées regroupent le WWF (Fonds mondial pour la nature), le CEFAID (Centre pour l'éducation, la formation et l'appui aux initiatives de développement), l'OPAD (Organisation panafricaine pour le développement), le ROSE (Réseau des ONG locales du SudEst-Cameroun) et le PERAD (Organisation pour la protection de l'environnement, la recherche et l'appui au développement). Elles se sont chargées de l'information, la sensibilisation, l'organisation des élections et la formation. Certaines ont également apporté un appui financier et joué un rôle d'appui-conseil auprès des entreprises forestières. Les représentants de l'administration locale, communale et traditionnelle ont quant à eux apporté leur caution légale et contribué à la mobilisation des populations. Enfin, les populations locales se sont impliquées dans l'élection des membres des CPF chargés de défendre leurs intérêts et droits dans la gestion des forêts du domaine permanent.

\section{Organisation des CPF}

\section{Situation réglementaire et statutaire}

Les enquêtes menées sur le terrain ont dénombré de 2 à 17 villages par CPF, avec une moyenne de 5. La majorité des CPF des deux départements ne possèdent pas de règlement intérieur (52\% dans la Boumba et Ngoko et $75 \%$ dans la Kadey). Le cas échéant, le processus d'élaboration n'a pas été participatif, les membres ayant été peu impliqués, comme ce fut le cas dans la Boumba et Ngoko. Dans la Kadey, en revanche, le règlement intérieur est le résultat de plusieurs réunions d'échange et le reflet de l'implication du CTFC. Neuf CPF sur les 16 que comptent la Boumba et Ngoko ont un statut juridique, tandis qu'aucun n'en possède dans la Kadey (figure 3).

\section{Représentativité}

L'analyse de la composition des bureaux des CPF dans les deux départements montre une nette domination des Bantous par rapport aux Bakas. Au sein du groupe bantou, on note une sous-représentation des femmes (87 membres sur 647 , soit $13,4 \%$ ) et leur quasi-exclusion dans le groupe baka (7 membres sur 647 , soit 1,1\%). Une meilleure représentativité de la minorité baka dans les bureaux des CPF est observée dans la Kadey, avec 63 membres sur 392 (soit 16,1 \%), contre 26 membres sur 249 (soit 10,4\%) dans la Boumba et Ngoko (figure 4). 


\section{CPF sans Règlement intérieur $\quad$ CPF sans statut}

$120 \%$
$100 \%$

$80 \%$

$60 \%$

$40 \%$

$20 \%$

$0 \%$

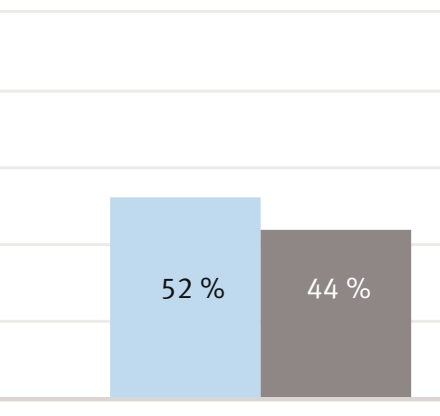

Boumba et Ngoko

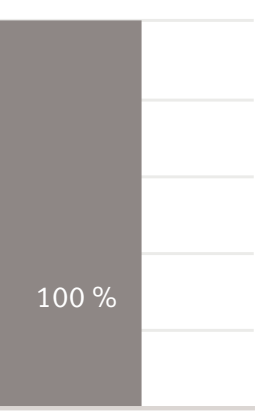

Kadey
Figure 3.

Situation réglementaire et statutaire des Comités paysans-forêts (CPF).

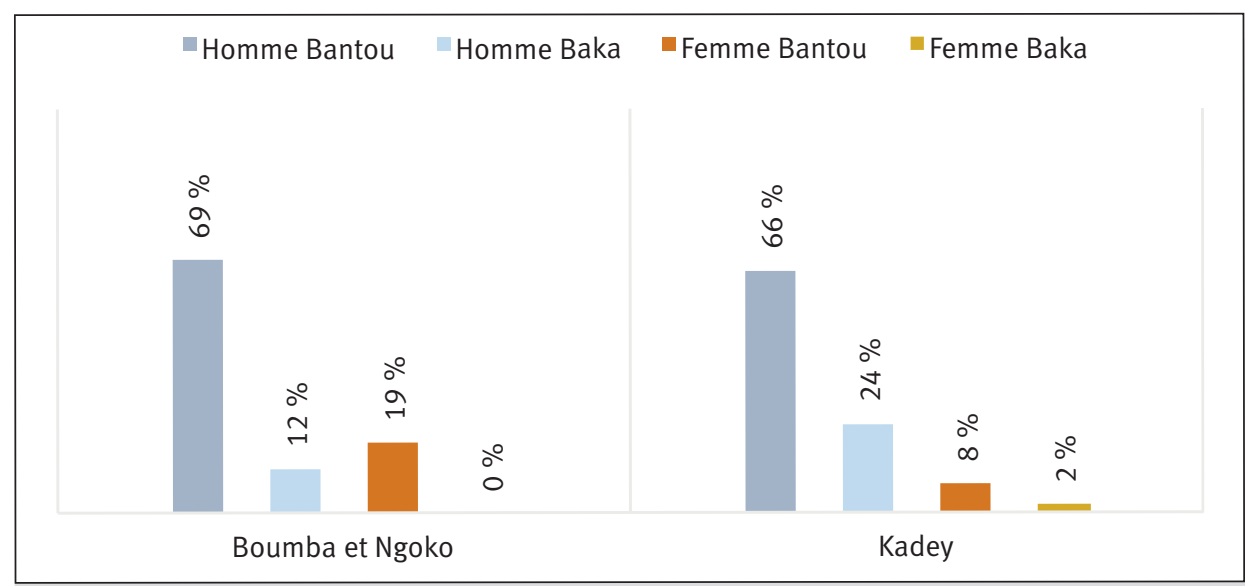

Figure 4.

Représentativité des différentes composantes sociales au sein des CPF.

Types d'activités d'animation et de sensibilisation

Très peu de CPF mènent des activités d'animation et de sensibilisation au sein de leur communauté. L'écoute et la retransmission des doléances des communautés (indicateur 1.4$)$ constituent la principale activité menée par les CPF (25\% dans la Boumba et Ngoko et $35 \%$ dans la Kadey) (figure 5). Les séances de discussion au sein des communautés ne sont pas non plus régulièrement organisées.

\section{Ressources financières}

L'enquête révèle que $44 \%$ des CPF de la Boumba et Ngoko et $71 \%$ de ceux de la Kadey ne disposent pas de ressources financières autonomes. Les quelques ressources enregistrées, orientées vers le fonctionnement des CPF, proviennent des activités génératrices de revenus telles que l'exécution des travaux d'aménagement (rafraîchissement et matérialisation des limites), la vente de plants (photo 1), la vente des rebuts de bois et le financement direct par le concessionnaire d'UFA, soit en moyenne 50000 francs CFA par an.

\section{Interaction entre CPF et autres acteurs}

Sur le terrain, deux modèles de collaboration ont été identifiés entre les CPF et les autres acteurs de la gestion forestière (administration forestière, ONG locales, communes). La collaboration formelle se caractérise

\section{Fonctionnement des CPF}

\section{Organisation des réunions et activités}

Pour avoir un fonctionnement cohérent, les CPF doivent tenir des réunions selon une fréquence régulière imposée par la réglementation, soit quatre réunions au moins par an (tableau II ; critère 6.1). Or, dans la pratique, le nombre maximum de réunions organisées est de trois par an, avec parfois une seule réunion annuelle. Par ailleurs, un seul CPF possède un plan d'action.

\section{Formation}

Les CPF ont chacun bénéficié d'au moins une séance de formation organisée par les structures ayant facilité leur mise en place. Les formations dispensées dans ce cadre ont porté sur neuf thématiques : rôle et mission du CPF, gestion durable des forêts, gestion et prévention des conflits, techniques d'animation et de sensibilisation en milieu rural, identification participative et montage des microprojets de développement, gestion des activités génératrices de revenus, utilisation du GPS, mise en place et entretien des pépinières, élevage des aulacodes (Thryonomys), pisciculture ou autre élevage. par la signature d'une convention ou un protocole d'accord entre le CPF et un autre acteur. Ce modèle de collaboration est uniquement observé chez certains concessionnaires d'UFA certifiées FSC (Forest Stewardship Council), telles la SEFAC (Société d'exploitation forestière et agricole du Cameroun) et la SFIL (Société forestière et industrielle de la Lokoundjé). Il se décline sous la forme d'un accord ou d'une convention qui définit les différentes obligations de chacune des parties. La « collaboration informelle ", modèle le plus rencontré, est utilisée par les concessionnaires d'UFA et les autres acteurs de la gestion forestière. Dans ce cadre, l'un des acteurs peut solliciter l'intervention de l'autre sans que cela fasse l'objet d'un engagement formel.

\section{Relations entre CPF et entreprises forestières}

Les niches de collaboration identifiées dans la législation sont peu expérimentées sur le terrain. Les entreprises forestières ne mettent pas à la disposition des CPF toutes les informations pertinentes relatives à la gestion de leurs concessions. Aucun des CPF de la Boumba et Ngoko ne possède le Plan d'aménagement (PA) du titre forestier duquel il est riverain, et seulement $25 \%$ des CPF de la Kadey en dispo- 
sent. En plus, ces derniers n'ont pas été impliqués dans le processus d'élaboration des PA et autres Plans de gestion forestière (PGF), la majorité d'entre eux ayant été mis en place après l'élaboration de ces documents. Seul le CPF dénommé Tikondi-Bélengou, riverain de l'Assiette annuelle de coupe (AAC) de l'UFA 10052 certifiée FSC, ouverte à l'exploitation, a effectivement participé à l'élaboration du plan annuel d'opération. Les CPF riverains de la FC de Batouri ont eux aussi participé au processus de classement de la forêt grâce à l'appui technique du CTFC.

Par contre, l'exécution des travaux en forêt par les concessionnaires d'UFA se pratique dans certains cas avec l'appui des CPF. Dans ce cas, deux modes d'arrangement sont observés. II peut s'agir de la signature d'un contrat de prestation rémunéré entre les CPF et les concessionnaires, disposition spécifiquement observée dans la Boumba et Ngoko mais fortement entravée par l'absence de statut juridique de certains CPF. L'arrangement peut aussi consister en la mise à disposition de la main-d'œuvre locale auprès des concessionnaires pour l'exécution des travaux ponctuels, ce type de collaboration étant fréquemment rencontré dans la Kadey. Lorsque le besoin en main-d'œuvre pour l'exécution de travaux ponctuels (rafraîchissement et matérialisation des limites, reboisement) (photo 2) en forêt est manifesté par le concessionnaire, ce dernier adresse une correspondance au président du CPF dans laquelle sont mentionnés le type de compétence recherchée, le groupe ethnique et éventuellement le village d'origine. Le président du CPF, le représentant du village au sein du CPF, le chef du village et les personnes proposées apposent leur signature sur la liste qui est transmise au concessionnaire.

\section{Relations entre CPF et chefs de postes forestiers}

La réalisation des activités de surveillance et de contrôle de l'exploitation forestière illégale suppose une étroite collaboration entre les CPF et les chefs de postes de contrôle forestier. Sur le terrain, respectivement 80 \% et $82 \%$ des CPF de la Boumba et Ngoko et de la Kadey ne mènent aucune activité conjointe avec les chefs de postes forestiers. Ceux qui travaillent conjointement avec les chefs de poste les accompagnent lors des missions de répression dans la Boumba et Ngoko et lors des patrouilles de contrôle dans la Kadey, cette collaboration restant guidée par la bonne volonté du chef de poste.

\section{Relations entre CPF, ONG locales et autorités administratives locales}

Une faible collaboration entre les CPF, les ONG, les autorités administratives et municipales est observée sur le terrain. Il est pourtant prévu que les CPF soient impliqués lors de l'élaboration des PA des FC. Seule la Cellule forestière communale (CFC) de la commune de Batouri dans la Kadey associe étroitement les CPF dans la mise en œuvre de ses activités.

$90 \%$

$80 \%$

$70 \%$

$50 \%$
Écoute et transmission Organisation des séances de discussion

$35 \%$

$30 \%$

$25 \%$

$20 \%$

$15 \%$

$10 \%$

$5 \%$

$0 \%$

$\quad 13 \%$

$35 \%$

$6 \%$
Kadey

Figure 5 .

Types d'activités d'animation et de sensibilisation menées par les Comités paysans-forêts (CPF).

$60 \%$

$40 \%$

$30 \%$

$20 \%$

$10 \%$ $0 \%$

1.1 1.21.31.41.52.12.22.33.13.23.34.14.24.34.44.55.15.25.36.1

-Boumba et Ngoko $\quad$ Kadey

Figure 6.

Niveau de conformité des indicateurs de performance chez les Comités paysans-forêts (CPF).

\section{Évaluation des écarts}

Sur la base de la décision $n^{\circ} 1354$, six critères d'évaluation et vingt indicateurs de performance ont été proposés pour évaluer le niveau de conformité des CPF avec les exigences légales. Il ressort de cette évaluation que neuf indicateurs $(1.5,2.1,2.2,2.3,3.1,4.1,4.4,5.2,6.1$; voir tableau II) sont non conformes pour tous les CPF, ceci dans

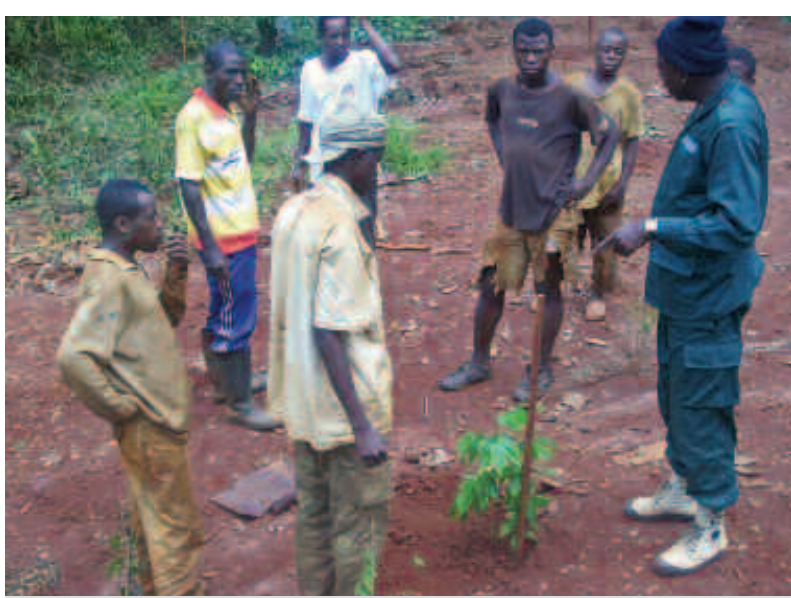

Photo 2.

Membres baka d'un Comité paysans-forêts qui effectuent le reboisement d'un parc en forêt.

Photo cellule d'aménagement de la société CFC/Thanry. 
Tableau III.

Pistes d'amélioration de l'efficacité des CPF.

Indicateurs

$1.1 ; 2.1$

$1.2 ; 1.4 ; 2.2 ;$

$2.3 ; 5.3$

$1.3 ; 4.5 ; 3.1 ;$

$3.2 ; 3.3$

$1.5 ; 4.1 ; 4.2 ; 4.4$;

$5.1 ; 6.1 ; 5.2$

4.3

\section{Pistes d'amélioration}

Multiplication des rencontres avec les communautés villageoises

Multiplier les canaux de diffusion des informations auprès des populations en profitant par exemple des rassemblements (jours de marché, cultes) pour organiser des séances de discussion.

\section{Amélioration de la collaboration entre les CPF et les autres acteurs de la gestion forestière} Favoriser la mise à la disposition des CPF des textes législatifs en matière de gestion forestière. Formaliser le cadre de travail CPF-MINFOF, les PTA (Plan de travail annuel) des postes forestiers doivent contenir des activités d'information des CPF en temps réel et les membres des CPF doivent être impliqués dans les missions conjointes de répression organisées par le chef de poste forestier. Développer un lien plus étroit avec les autorités traditionnelles et autres leaders d’opinion pour recueillir et transmettre à qui de droit les préoccupations des populations. Opérationnaliser les plates-formes de concertation pluri-acteurs afin de faciliter les échanges entre acteurs de l'exploitation forestière.

\section{Développement des mesures coercitives par le MINFOF}

Ces mesures permettront de rendre obligatoires : la mise à la disposition des CPF des résumés des PA des UFA et des FC; l'association des CPF à la réalisation des travaux de matérialisation ou de rafraîchissement des limites des AAC, des études socio-économiques, à l'élaboration des PGF ; l'identification des zones à hautes valeurs de conservation et la définition des mesures de protection.

\section{Renforcement des capacités des CPF}

Les capacités des CPF doivent être renforcées. Sur le plan technique : la collecte des informations; le suivi de la bonne exécution des PA; la mobilisation des ressources humaines; la surveillance des massifs forestiers; le contrôle et l'alerte. Sur le plan organisationnel : la tenue de réunions à des fréquences prévues par la réglementation.

\section{Amélioration du cadre juridique et institutionnel des CPF}

Clarifier le statut juridique des CPF pour qu'ils puissent : mieux défendre les intérêts des populations ; établir des contrats de prestation de services avec les concessionnaires d'UFA et les communes; diversifier les partenariats.

MINFOF : ministère des Forêts et de la Faune.

\section{Discussion et conclusion}

les deux départements. Quatre indicateurs $(1.3,3.2,3.3$, 5.1) dans la Boumba et Ngoko, et quatre autres (1.2, 4.3, 4.5 et 5.3) dans la Kadey, présentent également un niveau de conformité nul. Dans les deux départements, 13 indicateurs (soit $65 \%$ ) présentent un niveau de conformité nul chez tous les CPF.

Les sept autres indicateurs ne sont pas conformes dans tous les CPF. Dans les départements de la Boumba et Ngoko et la Kadey, respectivement trois $(4.2,4.3,4.5)$ et trois $(3.2,3.3,4.2)$ indicateurs sont conformes dans au moins $50 \%$ des CPF. Dans chacun des départements, quatre autres indicateurs présentent des niveaux de conformité inférieurs à $50 \%: 1.1,1.2,1.4$ et 5.3 pour la Boumba et Ngoko, et 1.1, 1.3, 1.4 et 5.1 pour la Kadey (figure 6).

Au terme de ces observations, des propositions de mesures visant à améliorer l'efficacité de ces CPF ont été formulées, comme présenté dans le tableau III.
Les enquêtes menées dans le cadre de cette étude ont concerné 33 CPF sur les 53 recensés, soit 16 pour la Boumba et Ngoko et 17 pour la Kadey. L'analyse de leur organisation met en évidence de nombreuses lacunes dont, d'une part, l'absence de règlement intérieur et de statut, qui sont des instruments normatifs indispensables accordant aux CPF une légitimité dans le processus de défense efficace des intérêts et droits des communautés qu'ils représentent, et, d'autre part, le manque de synergie d'action entre les différentes parties prenantes en relation avec les CPF. Les pouvoirs publics et les partenaires au développement exigent en effet que les organisations endogènes aient une reconnaissance légale et des statuts dûment enregistrés pour bénéficier d'appuis techniques et financiers. La majorité des CPF de notre zone d'étude n'est donc pas éligible à ces appuis. Cette lacune est fortement liée au vide juridique observé dans l'annexe de la décision n 1354/D/MINEF/CAB du 26 novembre 1999 qui 
n'apporte aucun élément de clarification sur le statut juridique des CPF. Cette décision recommande par ailleurs qu'il y ait, au sein de chaque village riverain d'un massif forestier, une structure représentative des communautés, soit un CPF par village. Cette prescription n'est pas respectée sur le terrain où les CPF sont créés sur des bases quelque peu subjectives, selon les facteurs liés à la proximité, par regroupement de villages voisins, ou socioculturels (langues et nature des rapports entre les villages). Le non-respect des dispositions légales est la conséquence du désengagement de l'État qui a abandonné aux concessionnaires et aux ONG le processus de création des CPF. Concessionnaires et ONG ont choisi de créer les CPF par regroupement de villages, allégeant ainsi les coûts financiers associés à ce processus.

Les structures représentatives des communautés locales sont le reflet de la diversité culturelle et sociale des populations qu'elles représentent. Dans le cas de cette étude, les Bantous et les Bakas d'une part, les hommes et femmes d'autre part, doivent avoir une représentation équilibrée. Ce n'est malheureusement pas ce qui a été observé sur le terrain, où se manifeste une forte prédominance des Bantous au détriment des Bakas et une sous-représentation des femmes en général et des femmes baka en particulier. La faible représentativité des peuples autochtones baka et des femmes au sein des organes de gestion forestière est une source de dysfonctionnement selon Ezzine de Blas et al. (2011) qui concluent que la prise en compte de l'hétérogénéité et de la variabilité des acteurs est un préalable à la réussite de la participation. Ce déséquilibre dans la représentativité hommes/femmes n'est pas propre à notre étude. Dans l'arrondissement de Yokadouma, département de la Boumba et Ngoko, Nzoyem (2007) a relevé une proportion de 12,7 \% de femmes dans les organes de gestion des ressources forestières et fauniques. Bandiaki et Tiani (2008) montrent que la proportion de femmes dans les instances dirigeantes mais aussi leur niveau de participation dans la prise de décision rurale, en contexte de gestion décentralisée des forêts, sont faibles au Cameroun et au Sénégal. Une autre sous-représentativité observée est celle des populations baka par rapport aux Bantous dans les bureaux des CPF. Ngo Babjeck (2011) a souligné la sous-représentativité des Bakas par rapport aux Bantous lors de l'analyse situationnelle des mécanismes de participation des populations autochtones vulnérables dans les processus de prise de décision au Cameroun. Assembe Mvondo (2005) constate lui aussi la non-représentativité des Bakas dans le comité consultatif de gestion de la forêt communale de Dimako. Des dispositions sont heureusement prises par certains concessionnaires pour pallier la marginalisation des populations autochtones. C'est le cas de la SFIL qui a mis en place deux CPF exclusivement composés de Bakas dans le cadre de la gestion de son UFA 10052, certifiée FSC. Cette approche d'intervention peut cependant contribuer à exacerber le sentiment de clivage au sein des communautés, entravant ainsi la cohésion sociale, elle-même gage d'une véritable gestion participative.

Au plan fonctionnel, aucun CPF n'organise les quatre réunions annuelles prescrites par la loi. Ces CPF ne disposent pas de sources pérennes de financement et n'ont bénéficié de formations qu'au moment de leur création. Pourtant, l'organisation fréquente des activités de renforcement des capacités des membres des comités locaux est requise pour leur permettre de pallier leurs lacunes et de mieux assumer leurs responsabilités (Mbairamadji, 2009). Par absence de formation, les membres des CPF restent en marge des évolutions récentes dans le secteur de la foresterie. Les CPF de notre zone d'étude ne disposent pas de plans de travail dûment élaborés, et les réunions sont imposées par les activités en cours ou impulsées de l'extérieur par les concessionnaires d'UFA, les ONG ou les cellules forestières locales. Les rencontres avec les concessionnaires sont plus fréquentes que les réunions des bureaux. Les revenus des CPF dépendent exclusivement des concessionnaires forestiers, ce qui ne facilite par leur indépendance et leur capacité à imposer leur point de vue et/ou à faire usage de moyens de pression lors de la revendication des droits des communautés qu'ils représentent.

La situation ainsi décrite laisse supposer que le processus de gestion durable encadré par une réglementation appropriée n'est pas effectif sur le terrain. Les CPF n'existent et ne fonctionnent que dans les zones où sont basées des sociétés forestières qui sont engagées ou souhaitent s'engager dans le processus de la certification. Comme la majorité des CPF sont mis en place par les exploitants forestiers, ils se trouvent de fait en position de faiblesse et ne peuvent pas par conséquent assurer une réelle efficacité et une durabilité à leurs actions. Cette inefficacité des CPF est relevée dans d'autres études réalisées au Cameroun (Ngono et Defo, 2005 ; Assembe Mvondo, 2006 ; Nguenang et al., 2007 ; Ngo Badjeck, 2011 ; Lescuyer et al., 2012).

Les CPF sont des structures représentatives des populations qui jouent le rôle d'intermédiaires et facilitent le dialogue, la consultation et la négociation entre les villageois et l'administration forestière et autres acteurs de la gestion forestière (la commune, les ONG, les organismes de conservations, les exploitants). La législation prévoit que les membres des CPF soient directement impliqués dans toutes les étapes de l'élaboration des Plans d'aménagement, qu'ils jouent un rôle très actif dans l'exécution des activités en forêt, qu'ils travaillent en étroite collaboration avec les chefs de postes forestiers et la commune dans les activités de surveillance et de contrôle des massifs forestiers. Tel n'est pas le cas sur le terrain et les inadéquations entre les prescriptions de la décision $\mathrm{n}^{\circ} \mathbf{1 3 5 4}$, et la réalité l'attestent. Treize indicateurs sur 20 (soit $65 \%$ ) présentent des niveaux de conformité nuls dans tous les CPF, dans les deux départements. Aucun indicateur ne présente un niveau de conformité de $100 \%$. La participation des CPF dans le processus de gestion des forêts reste donc faible dans les deux départements. Cette situation est la conséquence des rapports quasi inexistants entre les CPF et les autres acteurs de la gestion forestière, de la faible appropriation de leur mandat et des insuffisantes identifiées dans la disposition légale.

L'inefficacité des CPF peut également être attribuée aux approches adoptées par les différents acteurs tant dans la mise en place que dans l'accompagnement. Pour remédier à cette situation, le MINFOF (ministère des Forêts et de la Faune) a édité en partenariat avec le CTFC un guide pratique régissant la mise en place et l'accompagnement des CPF afin d'harmoniser les approches d'intervention. Malheureusement, ce document n'a pas été suffisamment vulgarisé auprès des cibles. 
En outre, l'exploitation forestière et toute activité qui lui est associée sont souvent perçues comme une potentielle source de revenus dans l'imaginaire populaire. Les activités des CPF étant considérées comme bénévoles, les populations ne s'y intéressent pas suffisamment. Il est souhaitable que l'État du Cameroun développe des mesures incitatives qui contribueront à accroître la motivation des populations. Le gouvernement pourrait par exemple instituer une ligne budgétaire destinée au fonctionnement des CPF dans le cadre des $10 \%$ de la redevance forestière annuelle destinée aux populations.

Les dysfonctionnements mis en évidence sont fortement préjudiciables à l'atteinte des objectifs assignés aux CPF mais aussi à la participation des communautés au processus d'observation indépendante des forêts dans le cadre des APV (Accords de partenariat volontaire). Les CPF évoluent sans véritable cadrage, de manière spontanée, ce qui ne leur permet pas d'être proactifs et de prendre des initiatives pertinentes. Il est par conséquent indispensable que ces lacunes soient traitées afin de permettre aux CPF d'optimiser leur efficacité. C'est toute l'importance que revêt la proposition de pistes d'amélioration, préalable indispensable pour leur participation effective à la gestion des forêts, qui se focalisent sur l'information, l'autonomisation et la formation.

\section{Références bibliographiques}

Assembe Mvondo S., 2005. Décentralisation des ressources forestières et justice environnementale : analyse des évidences empiriques du Sud-Cameroun. Law, Environment and Development Journal, $1 / 1$ : 35-49.

Assembe Mvondo S., 2006. Forestry income management and poverty reduction: empirical findings from Kongo, Cameroon. Development in Practice, 16 (1): 68-73. [Online] http://www.tandfonline.com/doi/abs/10.1080/09614520 500450867.

Bandiaky S., Tiani A., 2008. Genre, représentation et participation dans la prise de décision rurale en contexte de gestion décentralisée des forêts : Le cas du Cameroun et du Sénégal. Workshop on Forest governance and decentralization in Africa, 8-11 avril 2008, Durban, Afrique du Sud, 22 p.

Collas de Chatelperron P., 2005. Gestion participative des forêts de production au Cameroun. Bois et Forêts des Tropiques, 283 (1) : 51-63.

De Wasseige C., De Marcken P., Bayol N., Hiol Hiol F., Mayaux P., Desclée B., Nasi R., Billand A., Defourny P., Eba'a Atyi R., 2010. Les forêts du Bassin du Congo. État des forêts 2010. Luxembourg, Office des publications de l'Union européenne, $276 \mathrm{p}$.

Djeumo A., 2001. Développement des forêts communautaires au Cameroun : genèse, situation actuelle et contraintes. Réseau de Foresterie pour le Développement Rural, 25 (b) : 1-17.

Eba'a Atyi R., Lescuyer G., Ngouhouo Poufoun J., Moulendè Fouda T., 2013. Étude de l'importance économique et sociale du secteur forestier et faunique au Cameroun. Rapport final. Bogor Barat, Indonésie, Cifor, 278 p.
Ezzine de Blas D., Ruiz Perez M., Vermeulen C., 2011. Management conflicts in Cameroonian community forests. Ecology and Society, 16 (1): 8.

Lescuyer G., 2006. Formes d'action collective pour la gestion locale de la forêt camerounaise : organisations « modernes » ou institutions « traditionnelles » ? Vertig0, 6 (3). [Online] http://vertigo.revues.org/index8029.html.

Lescuyer G., Assembe Mvondo S., Essoungou J. L., Toison V., Trébuchon J.-F., Fauvet N., 2012. Logging concessions and local livelihood in Cameroon: from indifference to alliance? Ecology and Society, 17 (1): 7.

Mbairamadji J., 2009. De la décentralisation de la gestion forestière à une gouvernance locale des forêts communautaires et des redevances forestières au Sud-Est Cameroun. Vertig0, 9 (1). [Online] http://vertigo.revues.org/index8614.html.

Mvongo M., 2012. Gestion participative des forêts du domaine permanent : cas de l'implication des Comités paysans-forêts du Département du Haut Nyong dans la gestion des forêts. Mémoire, Université de Dschang, Cameroun, 58 p. Ngo Badjeck M. M., 2011. Analyse situationnelle des mécanismes de participation des populations autochtones vulnérables dans les processus de prise de décision au Cameroun. Living Earth Foundation et Fondation camerounaise de la Terre Vivante, Cameroun, 38 p.

Ngono L., Defo L., 2005. Implication des populations locales dans la gestion des ressources fauniques dans le Sud-Est Cameroun. Mécanisme, impacts, contraintes, opportunités et perspectives. WWF, Jengi forest program, Cameroun, $41 \mathrm{p}$. Nguenang G. M., Delvienne Q., Beligne V., Mbolo M., 2007. La gestion décentralisée des ressources forestières au Cameroun : les forêts communales après les forêts communautaires. 6e CEFDHAC, 20-22 novembre 2007, Libreville, Gabon, 7 p.

Nzoyem D. S. C., 2007. Implication des femmes dans les organes de gestion des ressources forestières et fauniques dans l'arrondissement de Yokadouma. Rapport de stage, appui à l'autopromotion de la femme de la Boumba et Ngoko. Yaoundé, Cameroun, Organisation néerlandaise de développement, $41 \mathrm{p}$.

Oyono P. R., 2005. Profiling local-level outcomes of environmental decentralizations: The case of Cameroon's Forests in the Congo Basin. Journal of Environment and Development, 14 (2): 1-21.

Topa G., Karsenty A., Megavand C., Debroux L., 2009. The Rainforests of Cameroon: Experience and Evidence from a Decade of Reform. Washington, DC, USA, The World Bank Group, 198 p. 